\title{
EFECTO DE LA FERTILIZACIÓN NITROGENADA TARDÍA EN ASPECTOS DE CALIDAD PANADERA EN TRIGO (TRITICUM AESTIVUM L.)
}

\author{
EFFECTS OF THE LATE NITROGENATED FERTILIZATION IN QUALITY \\ ASPECTS OF WHEAT BAKERY
}

\author{
Hernán Pinilla Quezada ${ }^{1}$; Luis Eduardo Herrera Floody ${ }^{2}$
}

\section{RESUMEN}

\begin{abstract}
Se evaluó el efecto de aplicaciones adicionales de N en Z-65, en el rendimiento, contenido de proteína, gluten húmedo y volumen de sedimentación del trigo. Las aplicaciones de urea foliar aumentaron significativamente $(\mathrm{P}<0,01)$ las variables de calidad, pero la aplicación de salitre sódico al suelo no tuvo efecto. Las aplicaciones de $\mathrm{N}$ como salitre sódico y urea foliar no tuvieron efecto significativo en el rendimiento. Las dos dosis totales de $\mathrm{N}$ no cambiaron el rendimiento y calidad del grano.
\end{abstract}

Palabras clave: Fertilización nitrogenada, Triticum aestivum L., calidad panadera trigo.

\begin{abstract}
The effect of additional applications of $N$ in Z-65 was evaluated in the grain yield, protein content, humid gluten and volume of sedimentation of wheat. The applications of foliar urea significantly increased $(P<0,01)$ the variables of quality, but the application of sodium saltpeter to the ground did not have effect. The applications of $N$ as sodium and urea saltpeter foliar did not have significant effect in the yield. The two total doses of $N$ did not change to the yield and quality of the grain.
\end{abstract}

Key words: Nitrogenated fertilization, Triticum aestivum L., wheat bakery.

\section{INTRODUCCIÓN}

Las aplicaciones de $\mathrm{N}$ después de espigadura no inciden en el rendimiento del trigo (Mellado, 1996), pero pueden elevar el contenido de proteína del grano (Stone, 1999; Brown, 2000; Seghezzo y Molfese, 2001; Keller y Fontanetto, 2001), determinando así la valorización y comercialización del trigo (Granger, 2002). Por tanto, el presente estudio pretende determinar el efecto de las aplicaciones adicionales de $\mathrm{N}$ en estado de antesis, Z-65, en las variables de calidad panadera del grano.

\section{MATERIALES Y MÉTODOS}

Este experimento se realizó en un suelo ultisol, en la IX Región, $38^{\circ} 71^{\prime} \mathrm{S}, 73^{\circ} 17^{\prime}$ O, y clima templado lluvioso con influencia oceánica. El ensayo tuvo seis tratamientos, incluidos los testigos y cuatro repeticiones. Las unidades experimentales, parcelas, tenían $2 \mathrm{~m}$ de ancho por $6 \mathrm{~m}$ de largo. El cultivar sembrado el 8 de agosto de 2003 fue Dollinco INIA, a una dosis de $180 \mathrm{~kg} \mathrm{ha}^{-1}$. El diseño experimental fueron parcelas divididas con un arreglo factorial $2 \times 3$ de tratamientos (T): T1 (testigo), $200 \mathrm{~kg}$

\footnotetext{
Universidad de La Frontera, E-mail: hpin@ufro.cl

Universidad de La Frontera, E-mail: herreral@ufro.cl
} 
$\mathrm{N} \mathrm{ha}^{-1}$ y 0 kg N ha- en Z-65; T2, $200 \mathrm{~kg} \mathrm{~N} \mathrm{ha}^{-1}$ y $20 \mathrm{~kg} \mathrm{~N}^{-1}$ salitre sódico en Z-65 (SS); T3, 200 $\mathrm{kg} \mathrm{N} \mathrm{ha}^{-1}$ y $20 \mathrm{~kg} \mathrm{~N} \mathrm{ha}^{-1}$ urea foliar en Z-65 (UF); T4 (testigo), $230 \mathrm{~kg} \mathrm{~N}^{-1}$ y $0 \mathrm{~kg} \mathrm{~N}^{-1} \mathrm{en} \mathrm{Z}^{-65}$; T5, $200 \mathrm{~kg} \mathrm{~N} \mathrm{ha}^{-1}$ y $20 \mathrm{~kg} \mathrm{~N}^{-1}$ salitre sódico en Z-65 (SS); T6, $200 \mathrm{~kg} \mathrm{~N}^{-1}$ y $20 \mathrm{~kg} \mathrm{~N} \mathrm{ha}^{-1}$ urea foliar en Z-65 (UF). Las aplicaciones de $\mathrm{N}$ en Z-25 y Z-31 fueron realizadas con Supernitro 25 en cobertera. Se utilizó una solución foliar al $21,75 \%$, obtenida al diluir $43,5 \mathrm{~kg}$ de urea en 200 $\mathrm{L}$ de agua, con lo cual se aplicó $20 \mathrm{~kg} \mathrm{~N} \mathrm{ha}^{-1}$. Se fertilizó a la siembra con una mezcla de $610 \mathrm{~kg}$ ha $^{-1}$ compuesta por $228 \mathrm{~kg}$ de Súper Fosfato Triple, $150 \mathrm{~kg}$ de Fosfato Monoamónico, $19 \mathrm{~kg}$ de Salitre sódico, $110 \mathrm{~kg}$ de Sulpomag, $93 \mathrm{~kg}$ de Nitrato de Potasio y $10 \mathrm{~kg}$ de Boronat. Las variables medidas fueron rendimiento de grano, contenido de proteína, gluten húmedo y volumen de sedimentación. Todas las mediciones fueron realizadas bajo los protocolos establecidos por la norma oficial chilena de calidad de trigo NCh513. Luego de verificar homogeneidad de varianza y distribución normal de los datos, se efectuó un análisis de varianza utilizando SPSS (2000). Las medias se compararon con la prueba de Tukey $(\mathrm{p} \leq 0,01)$.

\section{RESULTADOS Y DISCUSIÓN}

Rendimiento: No hubo diferencias significativas en rendimiento de grano $(\mathrm{p}>0,01)$ entre tratamientos comparados (Figura 1).
No se registraron diferencias de rendimiento entre dosis totales de $\mathrm{N}, 200$ y $230 \mathrm{~kg} \mathrm{~N} \mathrm{ha}^{-1}$, debido al alto suministro de nitrógeno por el suelo, el cual llegó a $120 \mathrm{~kg} \mathrm{~N} \mathrm{ha}^{-1}$, suficiente para satisfacer la demanda de cerca de $240 \mathrm{~kg} \mathrm{~N} \mathrm{ha}^{-1}$ para el rendimiento obtenido (Rodríguez, 1993). Las aplicaciones tardías de $\mathrm{N}$ no influenciaron el rendimiento del grano debido a que los componentes del rendimiento ya estaban definidos en etapas de desarrollo anteriores, excepto el peso del grano, el cual depende principalmente de la disponibilidad de agua. Los resultados concuerdan con los reportados por Keller y Fontanetto (2001), quienes señalaron que las aplicaciones tardías de $\mathrm{N}$ al estado de antesis no afectan el rendimiento del grano.

Evaluaciones de calidad: No se verificaron diferencias entre dosis totales de N, sin embargo, sí se registraron diferencias altamente significativas entre los tratamientos, al aplicar urea foliar en antesis en todas las variables de calidad evaluadas (Figura 2).

Los resultados concuerdan con los trabajos publicados por Barker (1995) y Kettlewell y Cooper (1991) quienes encontraron aumentos en el contenido de proteína a aplicaciones suplementarias de nitrógeno vía foliar, entre espigadura y antesis. Por otra, parte Brown (2000) verificó incrementos de dos puntos porcentuales, 13 a 15\%, en el contenido de proteína al adicionar $20 \mathrm{~kg} \mathrm{~N} \mathrm{ha}^{-1}$ en estados tardíos de desarrollo. Echeverría y Studdert (1998)

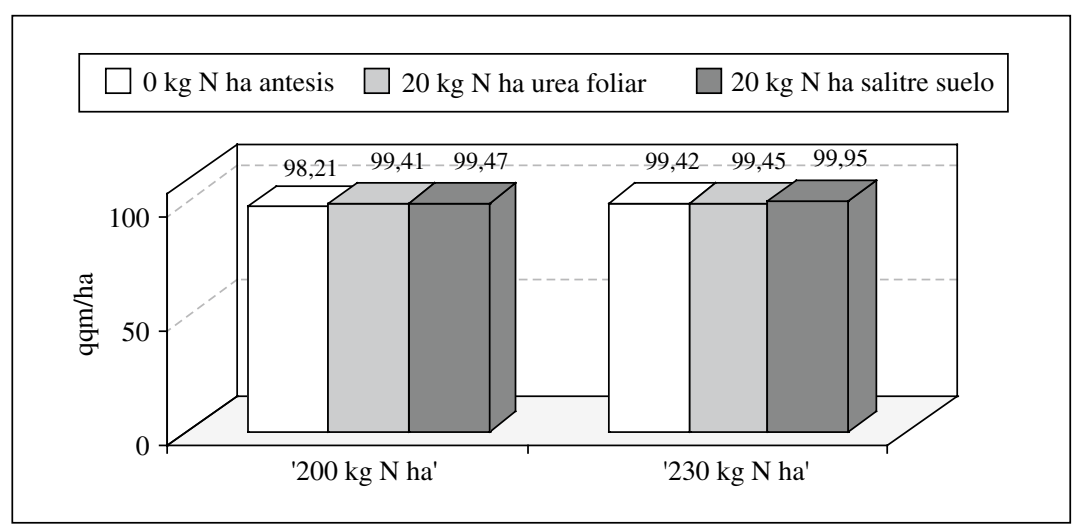

Figura 1. Rendimiento promedio de grano según tratamiento. 

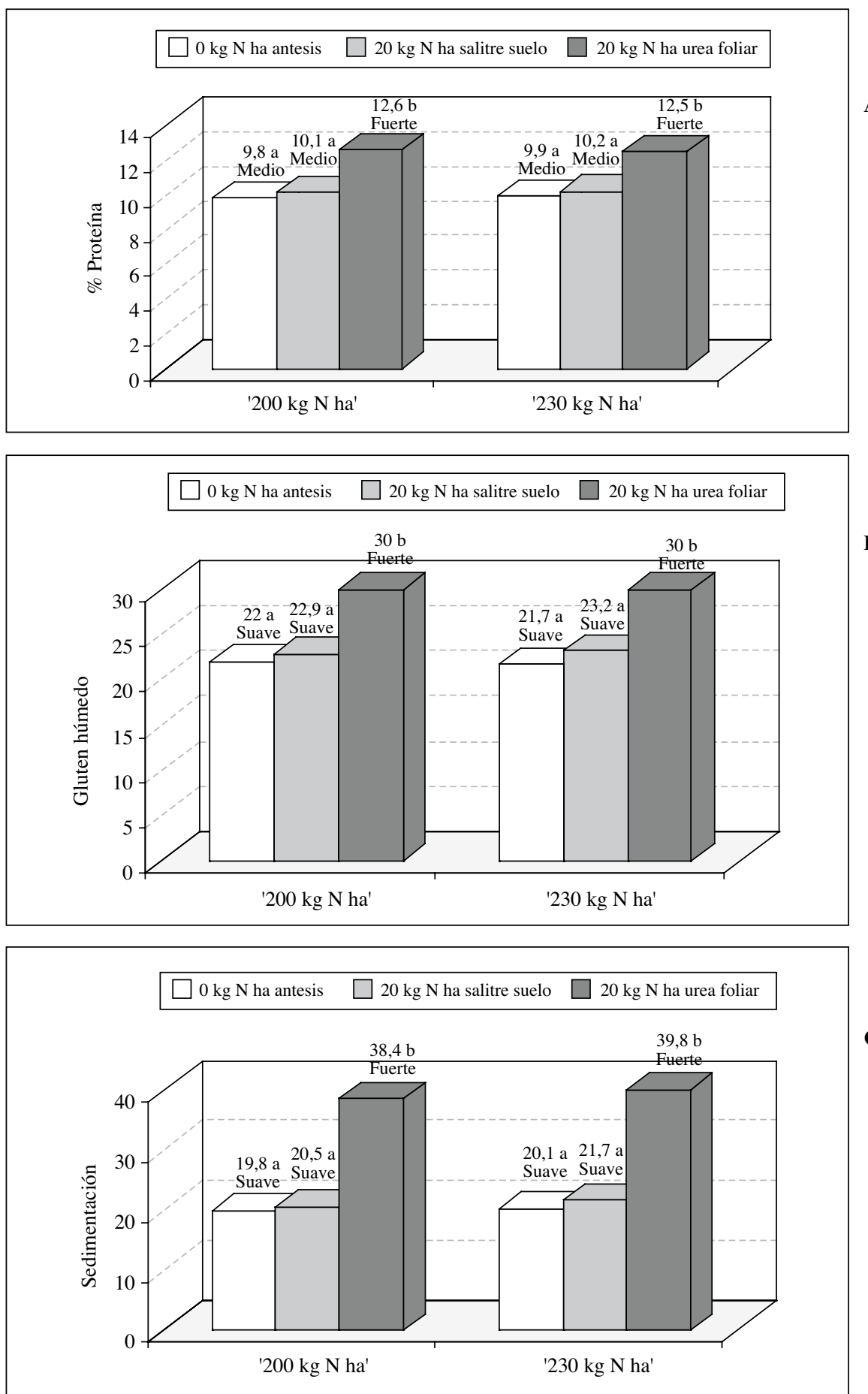

Figura 2. Efecto en las variables de calidad evaluadas y clasificación según tratamiento. A: proteína; B: gluten; C: sedimentación. *Cifras con letras distintas difieren estadísticamente según prueba de Tuckey, $(\mathrm{P}<0.01)$. 


\section{Cuadro 1}

Coeficientes de correlación Rho (r) de Spearman, entre las variables de calidad. ** significancia al 1\%

\begin{tabular}{|l|c|c|c|c|}
\cline { 3 - 5 } \multicolumn{2}{c}{} & Proteína & Gluten & Sedimentación \\
\hline Rendimiento & $\mathrm{R}$ & $0,16^{* *}$ & $0,06 * *$ & $0,12 * *$ \\
\hline Proteína & Significancia & 0,51 & 0,81 & 0,96 \\
\hline & $\mathrm{R}$ & 1 & $0,943 * *$ & $0,933 * *$ \\
\hline Gluten & Significancia & & 0,000001 & 0,000001 \\
\hline & $\mathrm{R}$ & $0,94 * *$ & 1 & $0,927 * *$ \\
\hline
\end{tabular}

encontraron que la aplicación de 20 y $40 \mathrm{~kg} \mathrm{~N} \mathrm{ha}^{-1}$ en espigadura incrementó el contenido de proteína entre 2,1 y 2,6 puntos porcentuales.

El contenido de gluten húmedo se elevó a niveles significativos por sobre el testigo, como lo afirman Bergh (2000) y Zamora et al. (2001), donde obtuvieron un aumento del contenido de gluten al aplicar $20 \mathrm{~kg} \mathrm{~N} \mathrm{ha}^{-1}$ y $40 \mathrm{~kg} \mathrm{~N} \mathrm{ha}^{-1}$ con urea en forma foliar.

La aplicación de urea foliar incrementó el volumen de sedimentación a valores de casi el doble del testigo. Kettlewell y Cooper (1991); Barker (1995) y Seghezzo y Molfese (2001) afirman que las aplicaciones de soluciones nitrogenadas foliares en estados tardíos de desarrollo, por ejemplo, con $30 \mathrm{~kg} \mathrm{~N} \mathrm{ha}^{-1}$ al estado de $50 \%$ de antesis, mostraron respuestas positivas en el volumen de sedimentación en trigos de invierno.

Asociación entre las variables de calidad: De acuerdo a los resultados presentados en el Cuadro 4, todas las variables de calidad evaluadas se correlacionaron significativa $(\mathrm{P}<0,01)$ y positivamente entre sí, lo que demuestra la existencia de una asociación muy alta entre estos y los factores de calidad.

\section{CONCLUSIONES}

1. Las aplicaciones suplementarias de $\mathrm{N}$ al estado de antesis no tuvieron efecto significativo sobre el rendimiento del grano.

2. Las aplicaciones de urea foliar al estado de antesis aumentaron significativamente todas las variables de calidad evaluadas respecto del testigo.

3. No se registraron diferencias significativas entre las dos dosis totales de $\mathrm{N}$ evaluadas con respecto al rendimiento, proteína, gluten húmedo y sedimentación.

4. La aplicación de salitre sódico al suelo no provocó efectos significativos sobre ninguna de las evaluaciones de calidad del grano.

5. Todas las variables de calidad se correlacionaron significativa y positivamente entre sí, mientras que no se correlacionaron con el rendimiento de grano.

6. En trigo, la aplicación de $\mathrm{N}$ en antesis vía urea foliar constituye una práctica agronómica factible de realizar debido a la alta respuesta que provoca en los parámetros de calidad, otorgando beneficios para el productor, la industria y el consumidor. 


\section{LITERATURA CITADA}

BARKER, B. 1995. How to grow high protein wheat. Agriculture and Agri-Food Canada Research Centre. $10 \mathrm{p}$.

BERGH, R. 2000. Fertilización nitrogenada para calidad en trigo candeal. CEI (Inta Tres Arroyos). Buenos Aires, Argentina. 90: 7-28.

BROWN, B. 2000. Increasing wheat protein. Nitrogen management for hard wheat protein enhancement. University of Idaho. Fertilizar facts $\mathrm{N}^{\mathrm{o}} 12$.

ECHEVERRÍA, S. Y STUDDERT, J. 1998. Fertilización y calidad en trigo. INTA Pergamino. Argentina.

FINCK, A. 1985. Fertilizantes y fertilización. Tendencias y métodos para la fertilización de cultivos. Editorial Reverte S. A., Barcelona, España. 439 p.

GRANGER, D. 2002. Contenido de proteínas y rendimiento por hectárea. Tierra Adentro (INIA). Temuco, Chile. 40: $32-33$.

INN. 2000. Instituto Nacional de Normalización-Chile (NCH 1237 - 2000). Requisitos de un trigo harinero. Primera edición. $16 \mathrm{p}$.

KELLER, O. Y FONTANETTO, H. 2001. Fertilización foliar en trigo. Publicación técnica campaña 2001, Nº15. INTA Rafaella. 14 p.

KETTLEWELL, P. AND COOPER, J. 1991. Nitrogen Fluids Improve Yield and Quality of Wheat. Soil-applied and foliar $\mathrm{N}$ applications show positive responses in UK winter wheat studies. Crop and Environment Research Center, UK. $125 \mathrm{p}$.

MELLADO, M. 1996. Aplicaciones basales y suplementarias de nitrógeno en trigo harinero (Triticum aestiuvum L.): Parte II, efecto sobre la calidad del grano. Agricultura Técnica (Chile). 56 (2): 122-127.

RODRÍGUEZ, J. 1993. La fertilización de los cultivos: un método racional. Colección en Agricultura, Facultad de Agronomía, Pontificia Universidad Católica de Chile, Santiago. 291 pp.

SEGHEZZO, M. L. Y MOLFESE, E. R. 2001. Calidad Comercial e industrial de Trigo. En: Trigo, Manual Técnico. Chacra Experimental Integrada Barro (Convenio MAG y AL-INTA): 83-88.

SPSS. 2004. Statistical Package for the Social Sciences (SPSS). SPSS Base 12.0 User's Guide for Windows. SPSS Inc., Chicago, Illinois, USA. Disponible en http://www.spss. com//spss.

STONE, S. 1999. Grain quality and its phisiological determinantes. En: Wheat Ecology and phisiology of yield and quality to short periods of post- antesis heat stress. Australian journal of Plant phisiology. 21: 887-900.

ZAMORA, M., SEGHEZZO, L. Y MOLFESE, E. 2001. Nutrición nitrogenada y proteína de trigo. Actas V Congreso Nacional de Trigo y III Simposio nacional de cereales de siembra otoño-invernal. $12 \mathrm{p}$. 
